\title{
Magicity quenching in neutron-rich nuclei and its consequences for electron-capture rates during core collapse
}

\author{
Ad. R. Raduta ${ }^{* a}$, F. Gulminelli ${ }^{b}$, M. Oertel $^{c}$ \\ ${ }^{a}$ IFIN-HH, P.O. Box MG-6, Bucharest-Magurele, Romania \\ ${ }^{b}$ CNRS/ENSICAEN/LPC/Université de Caen Basse Normandy, UMR6534, F-14050 Caen cédex, \\ France \\ ${ }^{c}$ LUTH, CNRS, Observatoire de Paris, Université Paris Diderot, 5 place Jules Janssen, 92195 \\ Meudon, France \\ E-mail: araduta@nipne.ro, gulminelli@lpccaen.in2p3.fr, \\ micaela.oertel@obspm. fr
}

\begin{abstract}
We study the impact of masses of very neutron-rich nuclei on matter composition and average electron capture rates during the late stages of pre-bounce evolution of core-collapsing massive stars. Inspired by experimental data which show magicity quenching in nuclei far from stability, we test the consequences of a possible quenching of shell closure in nuclei with $N=50$ and 82 . By using parametrized equations for electron-capture rates and empirical modifications of the DufloZucker mass formula, we show that magicity quenching in neutron-rich nuclei may increase the electron-capture rates by up to $30 \%$. Our results show the importance of new experimental data on nuclear masses and electron capture rates for neutron-rich nuclei in the vicinity of $N=50$ and $N=82$.
\end{abstract}

The Modern Physics of Compact Stars 2015

30 September 2015 - 3 October 2015

Yerevan, Armenia

${ }^{*}$ Speaker. 


\section{Introduction}

Nuclear physics is recognized to be an essential ingredient in astrophysics. The situation is very challenging as very often data away from nuclear matter saturation density, $n_{0} \approx 0.16$ $\mathrm{fm}^{-3}=2 \cdot 10^{14} \mathrm{~g} / \mathrm{cm}^{3}$, and isospin symmetry is required. This is in particular the case of neutron stars whose maximum mass is determined by exotic particle degrees of freedom and interaction potentials at several times $n_{0}$ and whose radii chiefly depend on the isovector properties of the equation of state (EoS).

Weak interaction rates are known to play a role in many processes like late stages of massive star evolution, thermonuclear and core-collapse (CC) supernovae, nucleosynthesis and energy generation in X-ray bursts, accreting neutron star crusts and neutron star mergers. A recent publication [1] has extensively investigated the impact of electron capture (EC) rates on core-collapse and early post-bounce phases of core-collapse supernovae. By systematically modifying the EC rates within the present experimental uncertainties, Sullivan et al. [1] have shown that the results are more sensitive to weak interaction rates than to the progenitor model or the EoS. In particular, EC rates are found to modify by $+16 /-4 \%$ the mass of the inner core at the time of shock formation and by $\pm 20 \%$ the peak of electron neutrino -luminosity during the deleptonization burst.

During core-collapse, thermodynamical conditions cover a very large range [2]. Typically, baryonic number density $n_{B}$ ranges from $10^{-10}$ to $10^{1} \mathrm{fm}^{-3}$, and the temperature $100 \mathrm{keV}<T<$ $100 \mathrm{MeV}$. The proton fraction $0<Y_{p}<0.7$, i.e. very neutron-rich states are populated. A genuine question is therefore to which extend limited nuclear physics data away from stability affects the astrophysical evolution of massive stars.

This is exactly the topic we aim to address in our contribution. More specifically, we shall investigate the sensitivity of average EC rates on nuclear masses and magicity quenching of neutron rich nuclei far from stability.

\section{Sub-saturated nuclear matter at finite temperature}

Pioneering models [3,4] for core-collapse supernovae matter are generalizations of zerotemperature models. Relying on the single nucleus approximation (SNA), they consider that subsaturated nuclear matter is composed of a unique representative heavy nucleus, a gas of unbound self-interacting nucleons and a gas of $\alpha$-particles generically accounting for light clusters. These models, publicly available, have been widely used in astrophysical simulations. Though, the use of a unique heavy cluster is expected to affect the EoS properties and, even more, the weak interaction rates.

Quite recently a number of $\mathrm{N}$ (uclear) S(tatistical) E(quilibrium) models has been proposed as alternative to model finite-temperature nuclear matter at sub-saturation densities $[5,6,7,8,9,10$, 11]. All these models depict nuclear matter as a mixture of light and heavy nuclei treated as an ideal gas and a uniform distribution of self-interacting nucleons. The basic ingredient which links the multiplicity per unit volume of a cluster defined by its mass $A$ and atomic $Z$ numbers to the single particle partition function is the nuclear physics counter part of the Saha equations.

The importance of including the whole distribution of heavy and light clusters was investigated in Ref. [12] performing spherically symmetric core-collapse supernova simulations based on 
general relativistic radiation hydrodynamics and three-flavor Boltzmann neutrino treatment. NSE EoS were used along with SNA ones, based on the same nuclear interaction. Systematic comparison of various thermodynamical and dynamical observables at different moments and distances from the star center shows that the treatment of in-homogeneous nuclear matter is as important as the nuclear interactions for supernova dynamics and the neutrino signal. The study is nevertheless incomplete as individual reaction rates were replaced, in the spirit of SNA, by a single rate on the most probable cluster, using the simplified Bruenn parametrization [13].

Predictions of various NSE models are confronted in Ref. [14] along different constant- $Y_{p}$ and constant- $n_{B}$ trajectories as a function of temperature. The authors show that practical choices done for the fragment definition, the properties of the gas of unbound nucleons and their interaction leave a trace on the chemical composition.

In order to test the validity of a specific NSE approach, an important requirement which should be fulfilled is the convergence of the distribution at low temperatures, towards the most probable cluster as predicted by the SNA approach. Ref. [11] addresses this issue. It is shown that the mean-field-inspired definition of nuclear clusters as systems composed of bound clusters (erepresentation) [15], obtained by subtracting the contribution of unbound nucleons from the total number of particles populating the Wigner-Seitz cell, guarantees by construction the convergence of the two approaches. With increasing temperature, the deviation between NSE and SNA increases and, quite remarkably, the average NSE cluster is closer to the unique SNA cluster than the most probable NSE cluster.

The results presented in this talk have been obtained within the extended NSE model of Ref. [11]. In the grandcanonical ensemble, the multiplicity per unit volume of a cluster of mass and atomic numbers $(A, Z)$ writes,

$$
\langle n(A, Z)\rangle_{\beta, \mu_{B}, \mu_{3}}=\omega_{\beta \mu_{B} \mu_{3}}(A, Z)=\exp \left[-\beta\left(F_{\beta}^{e}\left(A, \delta, \rho_{g}, y_{g}, \rho_{p}\right)-\mu_{B} A_{e}-\mu_{3} I_{e}\right)\right],
$$

where $F_{\beta}^{e}(A, Z)$ is the free energy of the $(A, Z)$-nucleus in e-representation,

$$
F_{\beta}^{e}(i)=E^{e}-T \ln V-T \ln c_{\beta}-\frac{3}{2} T \ln A_{e},
$$

expressed in terms of energy, mass and charge numbers in e-coordinates, $E^{e}\left(A, Z, \rho_{g}, \rho_{p}\right)=E^{v a c}-$ $\varepsilon_{g} A / \rho_{0}+\delta E_{\text {Coulomb }}, A_{e}=A\left(1-\rho_{g} / \rho_{0}\right), Z_{e}=Z\left(1-\rho_{p g} / \rho_{0 p}\right)[15] . \quad c_{\beta}=g_{\beta}\left(m T /\left(2 \pi \hbar^{2}\right)\right)^{3 / 2}$ comes from the integration over kinetic degrees of freedom and $g_{\beta}\left(A, \delta, \rho_{g}, \rho_{g p}\right)=\sum_{E^{*}} \exp \left(-\beta E^{*}\right)=$ $\int_{0}^{<S>} d E^{*} \rho_{A, \delta}\left(E^{*}\right) \exp \left(-\beta E^{*}\right)$ stands for the sum of the contributions of all excited states up to the average separation energy of a neutron(proton), $\langle S\rangle=\min (\langle S n\rangle,\langle S p\rangle)$. In the above equations $m$ stands for the nucleon mass, $V$ for the volume and $T=1 / \beta$ for the temperature. $\rho_{g}, \rho_{p g}$ and $\varepsilon_{g}$ correspond to the unbound nucleon gas total and proton density and energy density. For the applications considered here, the non-relativistic mean-field approach will be employed and the SLY4 [16] effective interaction will be used. $E^{v a c}$ denotes the cluster energy in vacuum and $\delta E_{\text {Coulomb }}$ is the Coulomb modification due to electron screening. $\rho_{0}$ represents the saturation density of asymmetric nuclear matter with bulk isospin asymmetry $\delta$, different from the global isospin asymmetry of the cluster $(1-2 Z / A)$. $\rho_{p g}$ corresponds to the proton density at $\rho_{0}, \rho_{p g}=\rho_{0}(1-\delta) / 2$. For more details, see Ref. [11]. 
As one may see in eq. (2.1), the average multiplicity per unit volume of a cluster $(A, Z)$ depends on nuclear structure data via the nuclear mass and level density. In order to be as realistic as possible, experimental masses [17] are used whenever data exist. When experimental data are missing, predictions of phenomenological (FRDM [18], DZ10 [19]) or microscopic (BsK22 [20]) mass models can be used. Predictions exist, by definition, up to the drip-lines. As shown in Ref. [21], bulk and surface in-medium modifications of cluster energetics are such that, when embedded in a nucleon gas, nuclear ground states might be very different from the ground states in vacuum. To account for exotic nuclei beyond the drip lines, mass tables have been supplemented by a SLY4compatible liquid drop model parametrization from Ref. [22]. Though, we stress that under the thermodynamical conditions explored in this work these nuclei are not populated with significant abundances.

Level densities are implemented according to Ref. [23] which offers parametrizations based on the most comprehensive set of experimental data available so far in the literature.

\section{Electron capture rates}

Aiming to highlight the role of nuclear masses far from stability in CC, we shall consider the EC rates as known and fixed quantities. Probably the best way to account for the available theoretical uncertainties in weak interaction rates is the one employed in Ref. [1]. That is, to use all existing data tables ranked according to the reliability and supplement the missing information by analytic formula fitted on microscopic data. To avoid spurious effects due to interpolation between table grid points and mismatch between table data and analytic formula, we opt for employing an analytic expression for all nuclear species and thermodynamical conditions. As in Ref. [1], we use the expression proposed by Langanke et al [24],

$$
\lambda_{E C}=\frac{\ln 2 \cdot \mathscr{B}}{K}\left(\frac{T}{m_{e} c^{2}}\right)^{5}\left[F_{4}(\eta)-2 \chi F_{3}(\eta)+\chi^{2} F_{2}(\eta)\right]
$$

hereafter referred to as L03. $\eta=\left(Q-\Delta E+\mu_{e}\right) / T, T$ represents the temperature and $m_{e}$ and $\mu_{e}$ denote the electron rest mass and chemical potential, respectively. $F_{i}(\eta)$ denotes the relativistic Fermi integral, $F_{i}(\eta)=\int_{0}^{\infty} d x x^{k} /(1+\exp (x-\eta))$. L03 corresponds to the threshold case of the pioneering equation proposed by Fowler, Fuller and Newman [25], where the structure factor log $<$ $f t>$ was replaced by the ratio between the typical Gamow-Teller plus forbidden matrix element $\mathscr{B}$ and the constant $K=6146 \mathrm{~s}$. In addition, the influence of excited states in the parent and daughter nuclei is approximately accounted for by a constant shift $\Delta E=E_{f}-E_{i}$. The values of $\mathscr{B}=4.6 \mathrm{MeV}$ and $\Delta E=2.5 \mathrm{MeV}$ used in this work are those proposed in Ref. [24]. They have been obtained by fitting large scale shell model calculations.

Fig. 1 investigates the performances of eq. (3.1) by confronting its predictions with those of different shell model [26, 27, 28], empiric [29] and QRPA [30] calculations for two arbitrarily chosen thermodynamical conditions as mentioned on the two panels. Different nuclei are represented by their $Q$-value for electron capture. At low temperatures and electron densities, structure effects are very important: $\log \lambda_{E C}(Q)$ covers a huge domain and important scattering exists between neighboring- $Q$ nuclei. Though different, the predictions of the four considered models show the same pattern and, on the figure scale, close values. At high temperatures and electron densities, 

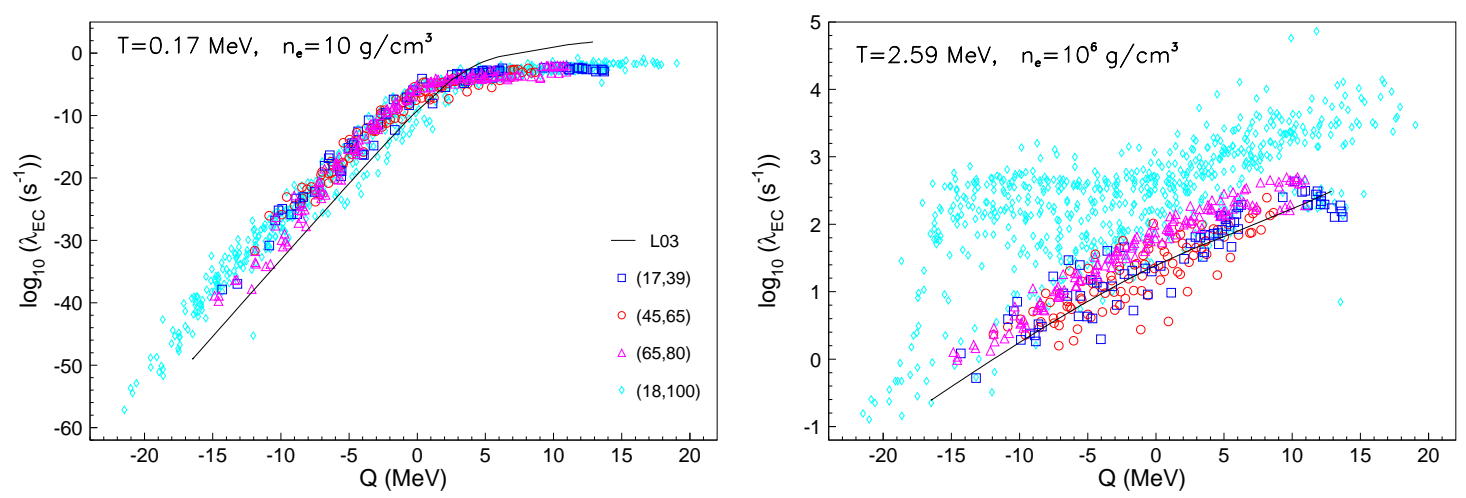

Figure 1: Electron capture rates: comparison between predictions of Eq. L03 and values of tables from Refs. [26] (17 $\leq A \leq 39)$, [27, 28] ( $45 \leq A \leq 65)$, [29] ( $65 \leq A \leq 80)$ and [30] $(18 \leq A \leq 100)$ for $\mathrm{T}=0.17$ $\mathrm{MeV}$ and $n_{e}=10 \mathrm{~g} / \mathrm{cm}^{3}$ (left panel) and, respectively, T=2.59 MeV and $n_{e}=10^{6} \mathrm{~g} / \mathrm{cm}^{3}$ (right panel).

structure effects are washed out: $\log \lambda_{E C}(Q)$ covers a much smaller domain and dispersion between neighboring- $Q$ nuclei is reduced. As mentioned in Ref. [24], the scattering of microscopic rates is an indication that several states with different transition strengths contribute to the same process. When the electron chemical potential is large enough such that EC becomes independent of the nuclear strength distribution, individual state structure is less important. Concerning the different models, we note that those of Refs. [26, 27, 28, 29] are in fair agreement while the QRPA calculations of Ref. [30] lead to systematically higher rates at high temperatures and electron densities. This behavior of QRPA rates has been discussed in Ref. [1], too. At high temperatures and electron densities, L03 is able to describe well the values of EC rates of Ref. [26, 27, 28, 29] even if deviations are obtained at low values of temperature and electron density. The over-estimations at positive $Q$-values are due to the truncated form of $\mathrm{L} 03$ with respect to the more general expression of Ref. [25]. We do not expect this to be a serious problem, since most of the nuclei produced during core collapse have negative $Q$-values. The underestimation at negative $Q$-values can be attributed to the constant values of $\mathscr{B}$ and $\Delta E$.

\section{Core-collapse trajectories}

To illustrate the consequences of experimentally unconstrained masses of neutron-rich nuclei, two core collapse trajectories are considered. They correspond to the pre-bounce evolution of the central element of two progenitors with zero age main sequence masses equal to $15 M_{\odot}$ and, respectively, $25 M_{\odot}$ as reported in Ref. [31].

Fig. 2 displays the temperature and electron fraction evolution as a function of time translated into baryonic number density. Upon collapsing, the central element becomes hotter and more neutron-rich. The $T\left(n_{B}\right)$ and $Y_{e}\left(n_{B}\right)$ patterns are the same for the two progenitors but the exact values are different. The more massive progenitor leads to higher temperatures and larger isospin asymmetries. The vertical bar on the $Y_{e}$-axis marks the domain where information on nuclear masses starts to be incomplete. 


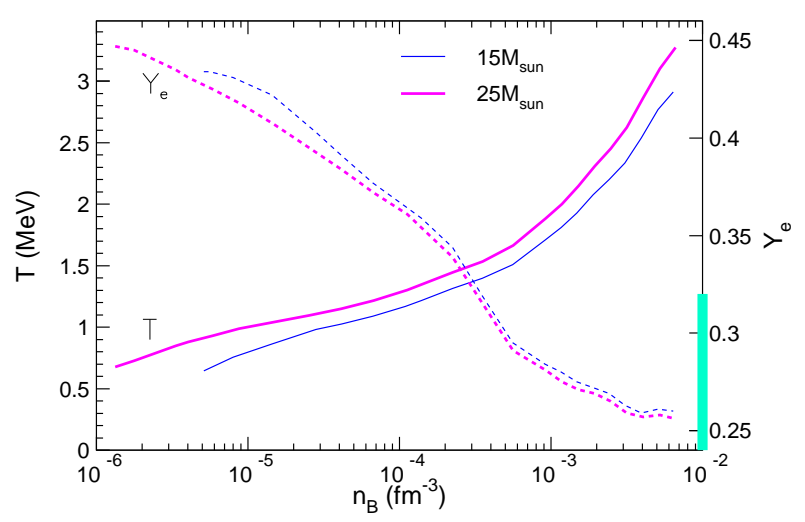

Figure 2: Thermodynamic conditions $\left(T, n_{B}, Y_{e}\right)$ spanned by the central element of two core-collapsing stars with $15 M_{\odot}$ and, respectively, $25 M_{\odot}$ as reported in Ref. [31]. Figure taken from Ref. [32].

\section{Chemical composition}

NSE cluster distributions obtained by using experimental masses supplemented with predictions of the DZ10-model [19] and the SLY4-LDM parametrization [22] show wide and complex shapes continuously evolving with $T, n_{B}$ and $Y_{e}$. Fig. 3 gives a first glimpse of fragment composition as a function of baryonic number density along the two trajectories in terms of mass fractions of unbound nucleons, light and heavy clusters. The two latter categories are arbitrarily defined with respect to $A=20$, where most of the bi- or multi-peaked mass distributions show a gap. One may see that heavy nuclei are dominant at any moment of the evolution though their importance decreases as the temperature increases. The mass fraction of unbound nucleons shows an overall increase with $n_{B}$. The mass fraction of light clusters shows a non-monotonic behavior and the strongest dependence on the progenitor mass. This non-monotonic evolution is the subtle consequence of the interplay between $T$ and $n_{B}$ which acts in opposite directions.

A deeper insight on chemical composition is given in Fig. 4. Each panel presents the $N$ and $Z$ numbers of the most probable and average heavy cluster. Vertical error bars correspond to the statistical dispersion of the distributions. Chemical composition of the central element of the $15 M_{\odot}$ progenitor is displayed on the left hand side while the one of the $25 M_{\odot}$ progenitor is shown on the right hand side. We may see that while the average proton number of heavy clusters is almost constant, the average neutron number increases monotonically with time. This is obviously due to the overall neutron-enrichment of stellar matter during collapse. The vertical bars show that, in addition to the average values, also the width of the $N$-distribution increases. By comparing the most probable with the average $N$ and $Z$ numbers, we note that they are never the same. This means that the distributions are not only broad, but also asymmetric. It is worthwhile to note that very often the most probable $N$ value is a magic number, 28 or 50 . The occurrence of $N$-magic numbers in different astrophysical sites has already been noted in the literature. In this context it due to the persistence of the stability valley magic numbers in neutron rich nuclei, according to DZ10 [19].

Indeed, Fig. 5 - that illustrates the two-neutron separation energy as a function of $N$-number along different isotopic chains copiously produced under the considered conditions - shows that gaps around $N=50$ and $N=82$ continue to be important in DZ10 even for extremely neutron- 


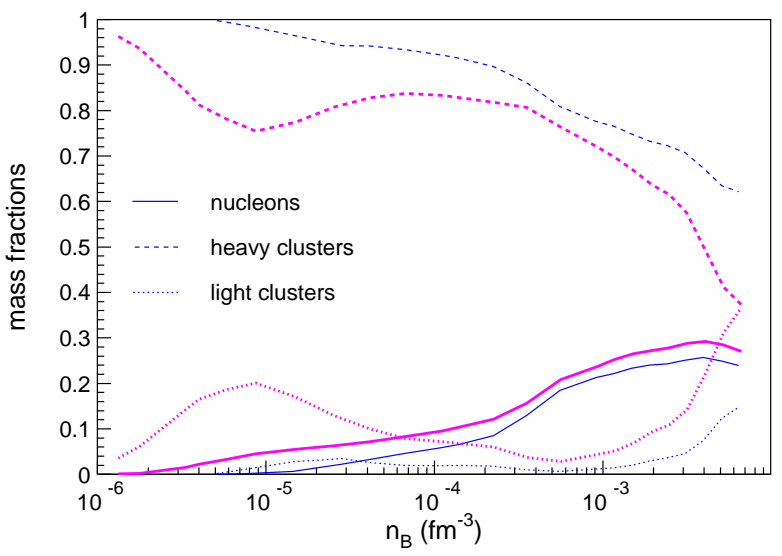

Figure 3: Mass fractions of free nucleons, light $(2 \leq A<20)$ and heavy $(A \geq 20)$ clusters obtained for the central element of the core-collapse with a $15 M_{\odot}$ (thin blue lines) and a $25 M_{\odot}$ (thick magenta lines) progenitor [31]. The temporal evolution is labeled via the baryon number density as in the previous figure. The color legend is the same as in the previous figure. Figure taken from Ref. [32].
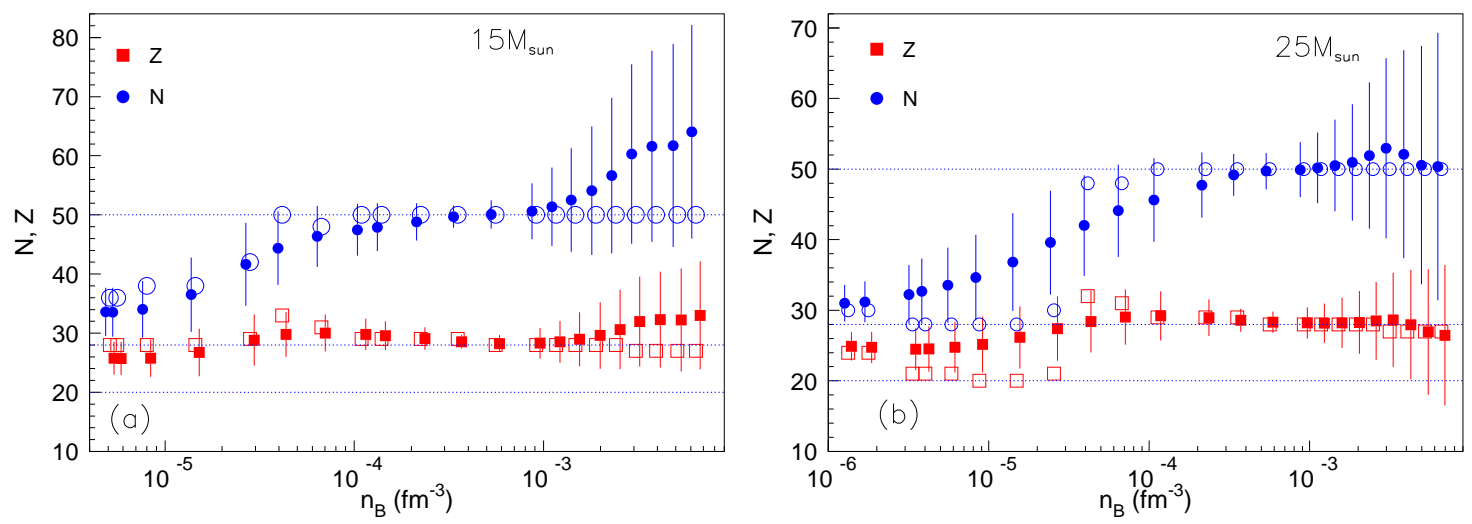

Figure 4: Average (solid symbols) and most probable (open symbols) proton and neutron numbers of heavy $(A \geq 20)$ nuclei produced in the central element of the $15 M_{\odot}$ and $25 M_{\odot}$ progenitors [31] during collapse as a function of baryonic density. The vertical bars correspond to the standard deviation of the distribution. For better readability, $N$ and $Z$ data have been slightly displaced in density. Figure taken from Ref. [32].

rich nuclei. At variance with this, the microscopic model BsK22 [20] manifests some magicity quenching for $N=50$. The situation is less clear for $N=82$ but, still, one can assume that magicity quenching can, in principle, occur here as well. Recent experimental data [33, 34] corresponding to $N=20$ magic number show that magicity quenching in neutron rich nuclei is to be expected. Indeed, while a huge gap is obtained for decreasing proton number up to ${ }^{34} \mathrm{Si}$, no gap is found for the even-even isotope ${ }^{32} \mathrm{Mg}$. This effect is due to both modification of single-particle energies far from stability and, more importantly, correlations which go beyond the single-particle shell model picture. Magicity quenching implies that very neutron-rich nuclei around $N=28,50,82$ will be less bound than if magicity survives. Eq. (2.1) shows that the multiplicity of a certain species depends, among other quantities, on its binding energy. As such, magicity quenching will 
lead to a decrease of nuclear abundances around $N=28,50,82$. In turn, in order to conserve $n_{B}$ and $Y_{e}$, nuclear species with neutron numbers situated between the magic numbers will be more copiously produced. As EC rates are convolutions of individual rates and the abundances of various species, $\left\langle\lambda_{E C}\right\rangle=\sum_{A, Z} \lambda_{E C}^{A, Z} n(A, Z) / \sum_{A, Z} n(A, Z)$ it is easy to anticipate that any modification of nuclear masses will impact the NSE-averaged reaction rates.

Consequences of magicity quenching in astrophysics have been previously addressed in Refs. $[35,36]$ in the context of r-processes. It has been shown that the discrepancy between canonical calculations in the regions $A \approx 110$ and $A \approx 180$ and experimental solar abundances is reduced.
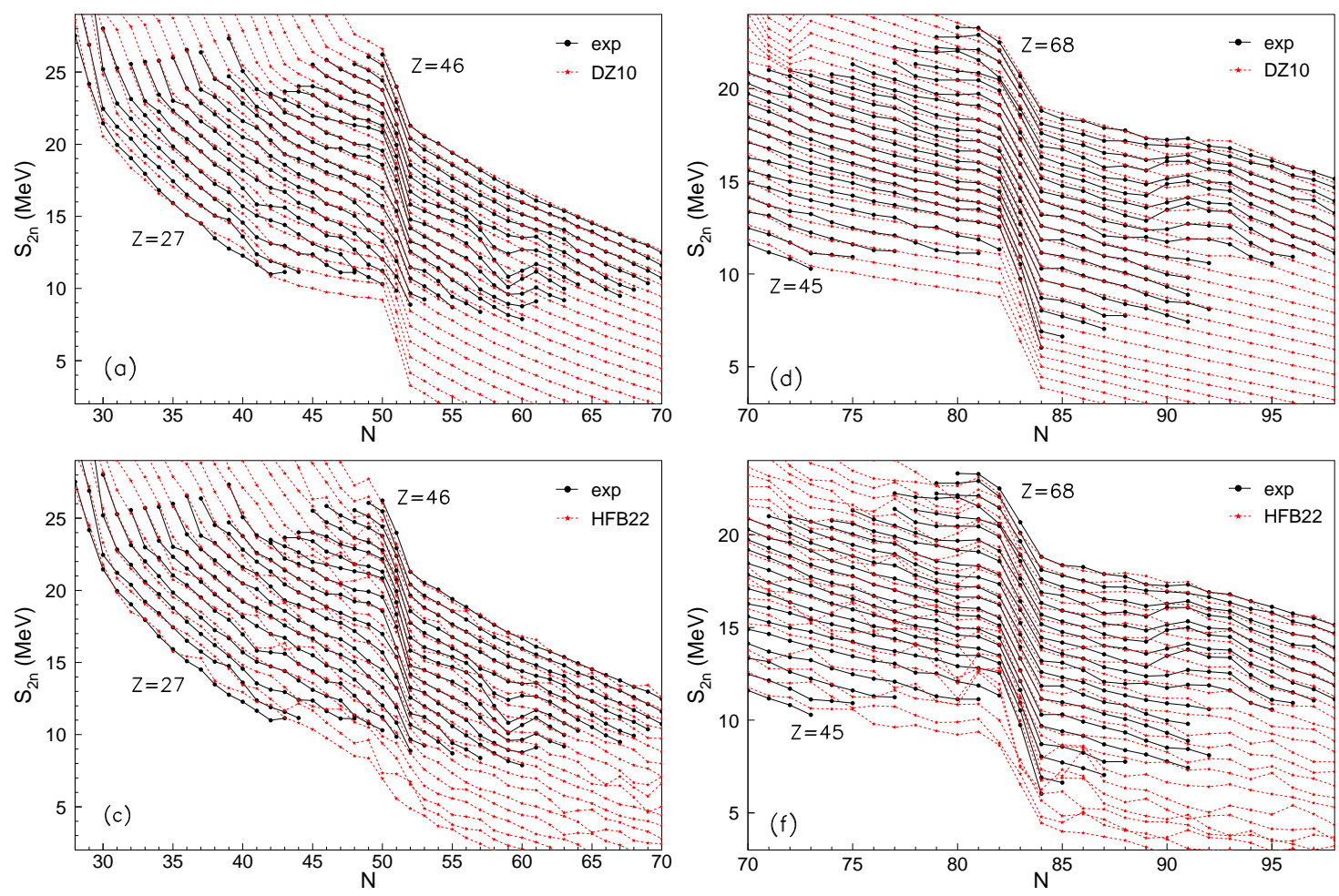

Figure 5: Two-neutron separation energy over selected isotopic chains as predicted by the DZ10 [19] and BsK22 [20] mass models (solid stars). Experimental data (solid circles) from Ref. [17] are plotted as well. Figure taken from Ref. [32].

\section{Evolution of magicity far from stability}

We follow the strategy proposed in Ref. [36] and introduce a modified expression of the binding energy,

$B^{m}(A, Z)\left\{\begin{array}{l|l}=B^{\exp }(A, Z), & Z_{i}^{\text {exp }}(A) \leq Z \leq Z_{s}^{\text {exp }}(A) \\ = & B^{L D}(A, Z)+f\left(Z_{i}^{\exp }(A)-Z, \Delta Z, \alpha\right)\left(B^{D Z}(A, Z)-B^{L D}(A, Z)\right), \\ Z_{i}^{D Z}(A) \leq Z<Z_{i}^{\text {exp }}(A) \\ =B^{L D}(A, Z)+f\left(Z-Z_{s}^{\text {exp }}(A), \Delta Z, \alpha\right)\left(B^{D Z}(A, Z)-B^{L D}(A, Z)\right), & Z_{s}^{\text {exp }}(A)<Z \leq Z_{s}^{D Z}(A),\end{array}\right.$ 
where $B^{\exp }(A, Z)$ and $B^{D Z}(A, Z)$ stand for the experimental binding energy [17] and predictions of DZ10 mass model [19], respectively. $Z_{i}^{\exp }(A)$ (resp., $\left.Z_{i}^{D Z}(A)\right)$ and $Z_{s}^{\exp }(A)\left(\right.$ resp. $\left.Z_{s}^{D Z}(A)\right)$ correspond to the most neutron-rich and, respectively, most neutron-poor nucleus with $A$ nucleons for which experimental masses (resp., predictions of DZ10) exist. $B^{L D}(A, Z)$ is a simple liquid-drop binding energy calculated according to

$$
B^{L D}(A, Z)=a_{v} A-a_{s} A^{2 / 3}-a_{v i} 4 I(I+1) / A+a_{s i} 4 I(I+1) / A^{4 / 3}-a_{c} Z(Z-1) / A^{1 / 3}+V_{p}(A, Z),
$$

with $I=|A-2 Z| / 2, a_{v}=15.62 \mathrm{MeV}, a_{s}=17.8 \mathrm{MeV}, a_{v i}=29 \mathrm{MeV}, a_{s i}=38.5 \mathrm{MeV}, a_{c}=0.7 \mathrm{MeV}$ and $V_{p}= \pm 12 / \sqrt{A} \mathrm{MeV}$ for even-even (+) and, respectively, odd-odd nuclei (-).
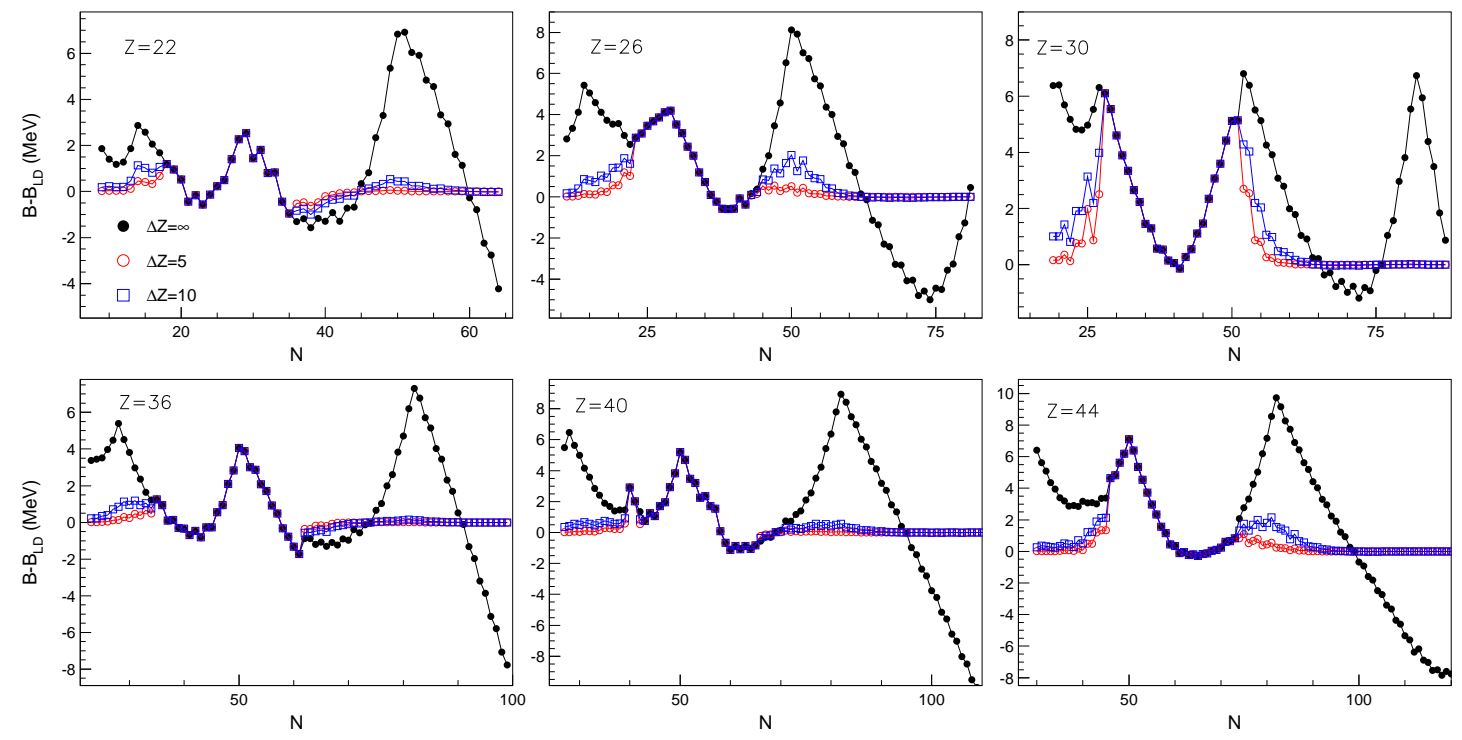

Figure 6: LDM-shifted binding energy as a function of neutron number for different isotopes strongly populated during core collapse. DZ10 [19] results (solid black dots) are plotted along modified results $B^{m}$ corresponding to two different scenarios of shell quenching. Figure taken from Ref. [32].

The arbitrary smearing function,

$$
f(x, \Delta Z, \alpha)=\exp [\alpha x / \Delta Z]
$$

depends on two parameters $\Delta Z$ and $\alpha<0$ which determine how fast the shell quenching occurs. Small values of $\Delta Z$ lead to a sudden quenching while the limit $\Delta Z \rightarrow \infty$ corresponds to nuclear masses described according to DZ10.

Fig. 6 illustrates the liquid drop shifted evolution of the modified binding energy introduced in eq. (6.1) as a function of neutron number for different isotopic chains of relevance for our study. Several values of $\Delta Z$ are considered, as mentioned on the figure. In all cases, $\alpha=\log \left(10^{-2}\right)$. As one may see, $B^{m}(A, Z)=B^{\exp }(A, Z)$ whenever experimental nuclear mass data exist. Outside the stability valley, $B^{m}(A, Z)$ smoothly evolves towards $B^{L D}(A, Z)$, which shows no structure effect. Peaks corresponding to magic numbers are suppressed faster for more exotic nuclei. 
The effect of the modification of the mass formula on the nuclear distribution is shown in Fig. 7 for two $\Delta Z$-values, as mentioned on the figure. Three thermodynamical conditions explored by the central element during the collapse of the more massive $25 M_{\odot}$ progenitor are considered. We note that as far as the baryonic matter has reduced isospin asymmetries and the most abundant nuclei lie in the stability valley, the modified $B^{m}(A, Z)$ binding energies do not produce any difference with respect to the DZ10 binding energy. On the contrary, at late stages in the pre-bounce evolution, where matter is neutron-rich, any modification of the binding energies results in a significant change of the produced species.
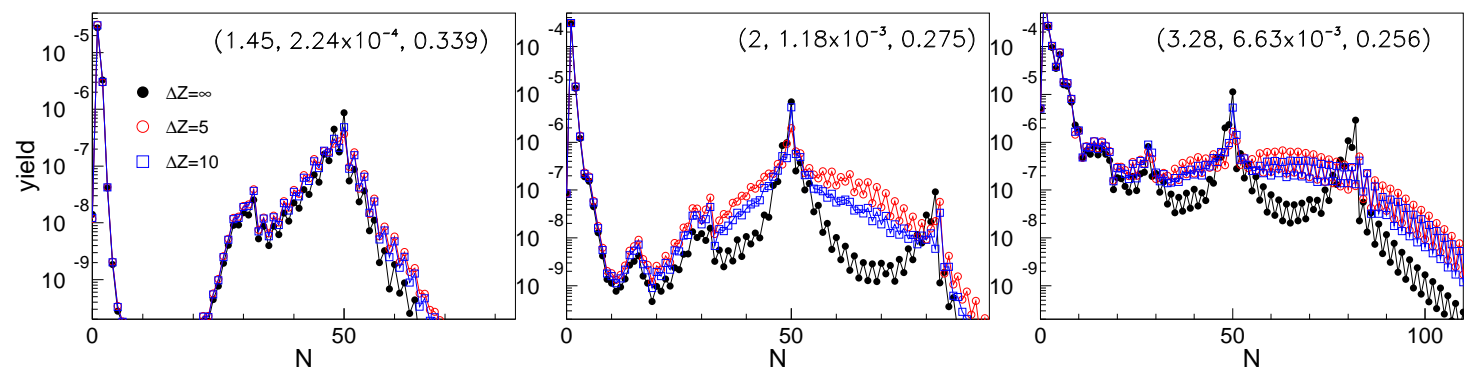

Figure 7: Impact of nuclear binding energies on nuclear abundances for three thermodynamic conditions $\left(T[\mathrm{MeV}], n_{B}\left[\mathrm{fm}^{-3}\right], Y_{p}\right.$ ) corresponding to different moments in the evolution of the central element of the collapse of a $25 M_{\odot}$ progenitor [31]. The distributions of clusters with a given neutron number are shown. The same prescriptions for the binding energies as in the previous figure are used. Figure taken from Ref. [32].

\section{Shell quenching and NSE averaged electron capture rates}

The main result of our investigation is depicted in Fig. 8. The consequences to different scenarios of shell quenching far from stability on NSE-averaged electron capture are plotted in terms of relative deviation with respect to the benchmark mass model DZ10. The values considered for the parameters of the smearing function are $\Delta Z=2,5,10$ and $\alpha=\log \left(10^{-2}\right)$. The results corresponding to each parameter set are plotted with a different color. Solid and dashed lines correspond to the collapse trajectories of the central element of the $15 M_{\odot}$ and, respectively, $25 M_{\odot}$ progenitors. The left panel represents the average EC rate on heavy $(A>20)$ nuclei. The right panel illustrates the inclusive effect of all species. Individual EC rates given by eq. (3.1) [24] are considered.

The pattern of both $\left\langle\lambda_{E C \text { heavy }}^{m}\right\rangle /\left\langle\lambda_{E C \text { heavy }}\right\rangle$ and $\left\langle\lambda_{E C}^{m}\right\rangle /\left\langle\lambda_{E C}\right\rangle$ is similar for the two collapse trajectories. The maximum deviation with respect to the fiducial model is obtained, as anticipated, at important neutron enrichment of matter provided that the temperature is small enough such that medium and heavy clusters are produced in a sizable amount. The most important message is that modifications in nuclear binding energies may lead to modifications of the total EC rates of more than 30\%. Similar calculations performed employing different EC parametrizations show that both the shape of $\left\langle\lambda_{E C \text { heavy }}^{m}\right\rangle$ and $\left\langle\lambda_{E C}^{m}\right\rangle$ and the amplitude of modification with respect to the fiducial model depend on the individual EC rates, as well. As such, it is of dramatic importance to better constrain nuclear masses and EC rates of neutron-rich nuclei by future experiments. 

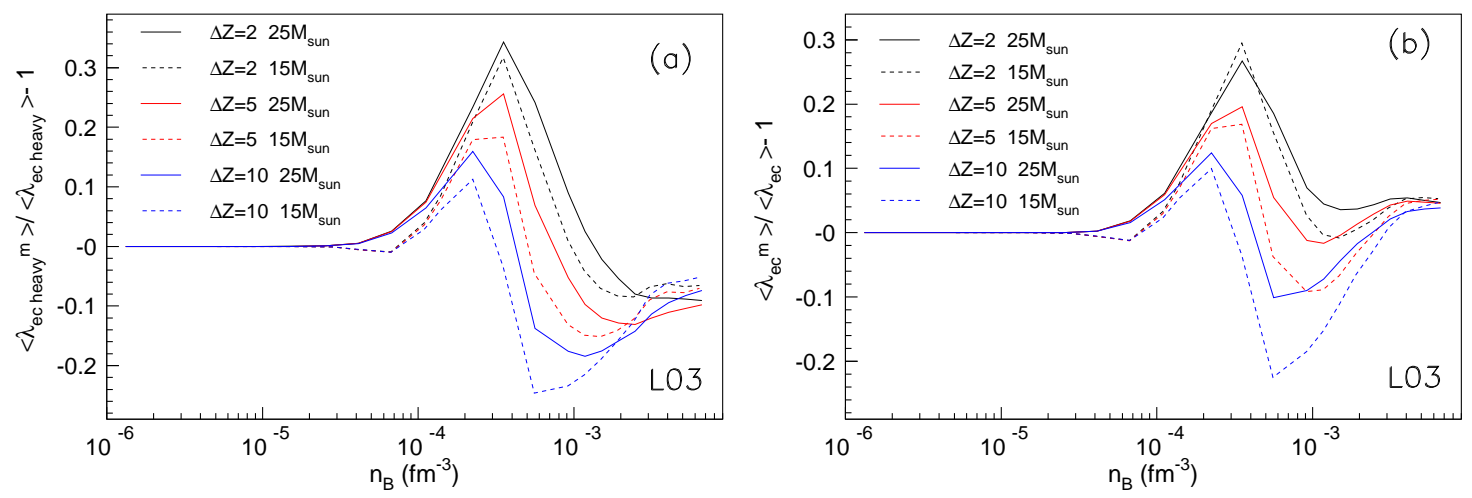

Figure 8: Ratio between NSE-averaged electron capture rates using the shell-quenched mass functional and the original DZ10 [19] mass model. The thermodynamic trajectories correspond to the central element of a $15 M_{\odot}$ (dashed lines) and a $25 M_{\odot}$ (full lines) core-collapsing progenitor [31]. The averaged rate is calculated only on heavy nuclei $(A \geq 20)$ in the left panel and on all nuclei in the right panel. Different quenching factors $\Delta Z$ are considered. For individual electron capture rates eq. (3.1) are considered.

\section{Conclusions}

We have examined the consequences of a possible quenching of neutron-magic numbers in very neutron rich nuclei on electron captures rates during core collapse. A recently developed extended NSE model [11] has been employed and typical thermodynamical conditions of the late stage of the pre-bounce evolution have been considered. They correspond to the central element of a $15 M_{\odot}$ and, respectively, a $25 M_{\odot}$ progenitors as reported in [31].

We have proven that a possible quenching of shell closures considerably affects the nuclear distribution and consequently the electron capture rates during the late stages of the collapse. The amplitude of the effect, measured with respect to the fiducial mass model of Duflo-Zuker [19], depends on the thermodynamical conditions, assumed scenario of shell quenching and the value of individual electron capture rates. The maximum value we have obtained when using the L03 parametrization of the electron-capture rates is of 30\%, meaning that important modifications in the astrophysical evolution can be expected.

We believe that additional experimental data of masses and electron capture rates of neutronrich nuclei around $N=50$ and 82 could be of great importance for a better understanding of the evolution of the collapse of massive stars at the end of their life.

\section{References}

[1] C. Sullivan, E. O'Connor, R. G. T. Zegers, T. Grubb, S. A. Austin, Astrophys. J. 816 (2016) 44.

[2] T. Fischer, M. Hempel, I. Sagert, Y. Suwa, J. Schaffner-Bielich, Eur. Phys. J. 50 (2014) 46.

[3] J. M. Lattimer, C. J. Pethick, D. G. Ravenhall and D. Q. Lamb, Nucl. Phys. A 432 (1985) 646; J. M. Lattimer and F. D. Swesty, Nucl. Phys. A 535 (1991) 331.

[4] H. Shen, H. Toki, K. Oyamatsu, and K. Sumiyoshi, Nucl. Phys. A 637 (1998) 435; H. Shen, H. Toki, K. Oyamatsu et al., Astrophys. J. Suppl. Series 197 (2011) 20. 
[5] S. R. Souza, B. V. Carlson, R. Donangelo, W. G. Lynch, A. W. Steiner, M. B. Tsang, Phys. Rev. C 79 (2009) 054602.

[6] S. Heckel, P. P. Schneider, A. Sedrakian, Phys. Rev. C 80 (2009) 015805.

[7] A. S. Botvina and I. N. Mishustin, Nucl. Phys. A 843 (2010) 98.

[8] M. Hempel and J. Schaffner-Bielich, Nucl. Phys. A 837 (2010) 210.

[9] Ad. R. Raduta and F. Gulminelli, Phys. Rev. C 82 (2010) 065801.

[10] S. I. Blinnikov, I. V. Panov, M. A. Rudzsky, K. Sumiyoshi, Astron. Astrophys. 535 (2011) A37.

[11] F. Gulminelli and Ad. R. Raduta, Phys. Rev. C 92 (2015) 055803.

[12] M. Hempel, T. Fischer, J. Schaffner-Bielich and M. Liebendorfer, Astrophys. J. 748 (2012) 70.

[13] S. W. Bruenn, Astrophys. J. Suppl. 58 (1985) 771.

[14] N. Buyukcizmeci et al., Nucl. Phys. A 90713 (2013).

[15] P. Papakonstantinou, J. Margueron, F. Gulminelli, and Ad. R. Raduta, Phys. Rev. C 88 (2013) 045805.

[16] E. Chabanat et al., Nucl. Phys. A 635 (1998) 231.

[17] G.Audi, M. Wang, A. H. Wapstra, F. G. Kondev, M. MacCormick, X. Xu, and B. Pfeiffer, Chinese Physics C 36 (2012) 1287; M. Wang, G. Audi, A. H. Wapstra, F. G. Kondev, M. MacCormick, X. Xu, and B. Pfeiffer, Chinese Physics C 36 (2012) 1603;

http://amdc.impcas.ac.cn/evaluation/data2012/data/nubase.mas12.

[18] P. Moller and J. R.Nix, Atom. Data Nucl. Data Tabl. 26 (1981) 165; P. Moller, J. R. Nix, W. D. Myers and W. J. Swiatecki, Atom. Data Nucl. Data Tabl. 59 (1995) 185.

[19] J. Duflo and A. P. Zuker, Phys. Rev. C 52 (1995) R23; http://amdc.in2p3.fr/web/dz.html.

[20] S. Goriely, N. Chamel, and J. M. Pearson, Phys. Rev. C 88 (2013) 024308.

[21] Ad. R. Raduta, F. Gulminelli, and F. Aymard, Eur. Phys. J. A 50 (2014) 24.

[22] P. Danielewicz and J. Lee, Nucl. Phys. A 818 (2009) 36.

[23] T. von Egidy and D. Bucurescu, Phys. Rev. C 72 (2005) 044311; 73 (2006) 049901(E).

[24] K. Langanke et al., Phys. Rev. Lett. 90 (2003) 241102; K. Langanke and G. Martinez-Pinedo, Rev. Mod. Phys. 75, (2003) 818.

[25] G. M. Fowler, W. A. Fuller, M. J. Newman, Astrophys. J. 293 (1985) 1.

[26] T. Oda, M. Hino, K. Muto, M. Takahara, K. Sato, Atomic Data and Nuclear Data Tables 56 (1994) 231.

[27] K. Langanke and G. Martinez-Pinedo, Nucl. Phys. A673 (2000) 481.

[28] K. Langanke and G. Martinez-Pinedo, Atomic Data and Nuclear Data Tables 79 (2001) 1.

[29] J. Pruet and G. M. Fuller, Astrophys. Journal Suppl. Series 149189 (2003).

[30] J.-U. Nabi, H. V. Klapdor-Kleingrothaus, Atomic Data and Nuclear Data Tables 71 (1999) 149; ibid. Atomic Data and Nuclear Data Tables 88 (2004) 237476.

[31] A. Juodagalvis, K. Langanke, W. R. Hix, G. Martinez-Pinedo, J. M. Sampaio, Nucl. Phys. A 848 (2010) 454.

[32] Ad. R. Raduta, F. Gulminelli and M. Oertel, Phys. Rev. C 93 (2016) 025803. 
[33] O. Sorlin, and M.G. Porquet, Prog. Part. Nucl. Phys. 61 (2008) 602.

[34] A. Chaudhuri et al., Phys. Rev. C 88 (2013) 054317.

[35] B. Chen, J. Dobaczewski, K.-L. Kratz, K. Langanke, B. Pfeiffer, F.-K. Thielemann, P. Vogel, Phys. Lett. B 355 (1995) 37.

[36] J. M. Pearson, R. C. Nayak, S. Goriely, Phys. Lett. B 387 (1996) 455. 